\title{
Éditorial
}

\section{P. Vautravers}

(C) Springer-Verlag France 2011

Les thérapeutiques manuelles pratiquées sous divers aspects et vocables (médecine manuelle, ostéopathie, chiropraxie...) font en France et en Europe l'objet d'une popularité croissante auprès du grand public, ce qui a conduit les gouvernements successifs à autoriser la pratique de ces thérapeutiques non seulement aux médecins mais également aux kinésithérapeutes, non-médecins et non-kinésithérapeutes.

L'engouement du public pour ces traitements est important en raison de leur efficacité antalgique remarquable. Il s'agit d'une thérapeutique non médicamenteuse de la douleur que le monde « scientifique et universitaire » commence à peine à découvrir. Il manque, en effet, des preuves de haut niveau pour pouvoir en asseoir définitivement l'efficacité.

Les thérapies manuelles, avec thrust ou sans thrust, sont enseignées aujourd'hui dans 15 facultés de médecine française dans le cadre d'un enseignement universitaire médical. Depuis une réglementation récente, des écoles privées, non universitaires, enseignent cette pratique aux non-médecins. Les champs d'application classiques des manipulations vertébrales et ostéoarticulaires concernent le rachis, les lombalgies, dorsalgies et cervicalgies mécaniques dites communes et la ceinture pelvienne dont l'articulation sacro-iliaque ; les algies pelvipérinéales d'origine gynécologique, digestive, urinaire ou neurologique, les coccygodynies, les douleurs périnéales du cycliste..., la sphère odontognathique, l'appareil manducatoire constituent des indications plus rares des thérapeutiques manuelles à la condition de les intégrer dans un protocole thérapeutique coordonné, médicochirurgical.

Nous remercions les intervenants qui apportent leur expérience, leur savoir-faire dans un domaine très complexe. Nous espérons qu'en médecine physique et réadaptation, rhumatologie, médecine manuelle, ostéopathie, médecine générale, médecine du sport et kinésithérapie... cette revue pourra apporter une référence de sérieux et de transparence ainsi qu'une contribution utile en pratique quotidienne.

P. Vautravers $(\bowtie)$

Service de médecine physique et de réadaptation,

hôpitaux universitaires de Strasbourg, CHU-Hautepierre,

F-67098 Strasbourg cedex, France

e-mail : Philippe.Vautravers@chru-strasbourg.fr 